\title{
Real-time correction method for image distortion on smart phone Chen Lei ${ }^{1, a}$, Tian Hengda ${ }^{1, b}$ and Ji Xiaoyong ${ }^{1, c}$ * \\ ${ }^{1}$ School of Electronic Science and Engineering, Nanjing University, Nanjing, 210023, China aclnjuee2011@163.com, ${ }^{\text {btianhengdanjupt@163.com, }{ }^{c} \text { ixy@nju.edu.cn }}$
}

Keywords: Mobile phone platform, image distortion, correction matrix

Abstract. To correct the trapezoidal distortion of images taken by mobile phone on the smartphone platform, a real-time correction method for image distortion based on correction matrix is proposed. First, the correction matrix data in various cases is calculated and stored in the database based on the control point method. Then, the application downloads the corresponding correction matrices and stores them in the mobile phone when starts for the first time, and invokes them directly to correct the distorted image captured in real time. Experimental results show that this method can quickly corrects the distorted images, and meets the expected functional requirements.

\section{Introduction}

With the development of artificial intelligence, smart phone, as the most popular personal computing platform, is bound to become the most important and convenient intelligent terminal for everyone, but as it brings great convenience to people's life and work, it also raises some worrying problems. For example, pedestrians play their mobile phones while walking, which will distract attention and lead to fall and injure, even car accidents, such incidents are constantly emerging in various news reports at home and abroad. In view of the above situation, an application can be run on the smart phone, and provide security protection for the pedestrians is particularly urgent ${ }^{[1]}$. The core is to collect the image information of the road ahead using the mobile phone camera in real time ${ }^{[2]}$, and then use the identification algorithm operating on the background to identify the pavement features and recognize the hidden danger to avoid the occurrence of tragedy. To develop such software, the following key technical issues need to be addressed:

1. When a mobile camera is shot at a certain angle, the resulting image will be distorted;

2. The computing power of intelligent terminal is limited, so it is difficult to meet the need of real-time fast correction of high-resolution images;

3. The software can avoid the underlying drivers and instead correct the captured images directly at the application layer;

In the above background, this paper proposes a real-time correction method for image distortion based on the correction matrix. The correction matrices corresponding to various shooting angles are captured and stored in the mobile phone, which can be invoked directly to correct the distorted image captured in real time. This method solves the problem of limited computing power of mobile phone platform, and improves the real-time performance of image correction.

\section{Real-time Correction Method for Image Distortion Based on the Correction Matrix}

Calculate the Correction Matrix. The image correction algorithm proposed in this paper is based on the control point method ${ }^{[3]}$. The detailed calculation process is as follows:

Step 1: Draw a large enough standard grid on the plate for standard test images.

Step 2: Fix the phone to the test platform, adjust to a specific height, and adjust the rotation angle of the $y$ and $x$ axis to the desired value. At this point, the height of the mobile phone, the rotation angle of the phone's $x$ and $y$ axis relative to the horizontal position is fixed and known ${ }^{[4]}$. We will discuss the numerical determination of the rotation angle in the $\mathrm{y}$ - axis and $\mathrm{x}$ - axis later.

Step 3: Use the API provided by the mobile phone system, invoke the camera control program, and collect a frame image in real time.

Step 4: Pretreat the collected images by graying and histogram equalization in $\operatorname{turn}^{[5]}$. The purpose of gray-scale is to reduce the time required for image processing. The purpose of histogram equalization 
is to make the edge contour feature clearer to get the control point on the distorted rectangle contour conveniently.

Step 5: Select four vertices of a distorted square contour on the standard test image after histogram equalization through human-computer interaction. To achieve a better correction effect, the square here should select the largest complete square that can be found in the image ${ }^{[6]}$. Click in the order of top-left, upper-right, left-lower, right-lower and store the coordinates of the vertices in the program. The obtained distortion contour vertices are the control points ${ }^{[7]}$, we set them as $\mathrm{A}\left(x_{a}, y_{a}\right), B\left(x_{b}, y_{b}\right), C\left(x_{c}, y_{c}\right), D\left(x_{d}, y_{d}\right)$, and the coordinates of the corresponding points in the corrected image are $E\left(x_{e}, y_{e}\right), F\left(x_{f}, y_{f}\right), C^{\prime}\left(x_{c}^{\prime}, y_{c}^{\prime}\right), D^{\prime}\left(x_{d}^{\prime}, y_{d}^{\prime}\right)$, as shown in fig. 1. The coordinates of the standard points $E F C^{\prime} D^{\prime}$ in the figure are calculated by the following algorithm:

$$
\begin{aligned}
& \left(x_{e}, y_{e}\right)=\left(x_{c}, y_{c}-L\right) . \\
& \left(x_{f}, y_{f}\right)=\left(x_{d}, y_{c}-L\right) . \\
& \left(x_{c}^{\prime}, y_{c}^{\prime}\right)=\left(x_{c}, y_{c}\right) . \\
& \left(x_{d}^{\prime}, y_{d}^{\prime}\right)=\left(x_{d}, y_{c}\right) .
\end{aligned}
$$

$L$ is the horizontal pixel difference between $\mathrm{C}$ and $\mathrm{D}$ points in the image:

$$
L=x_{d}-x_{c}
$$

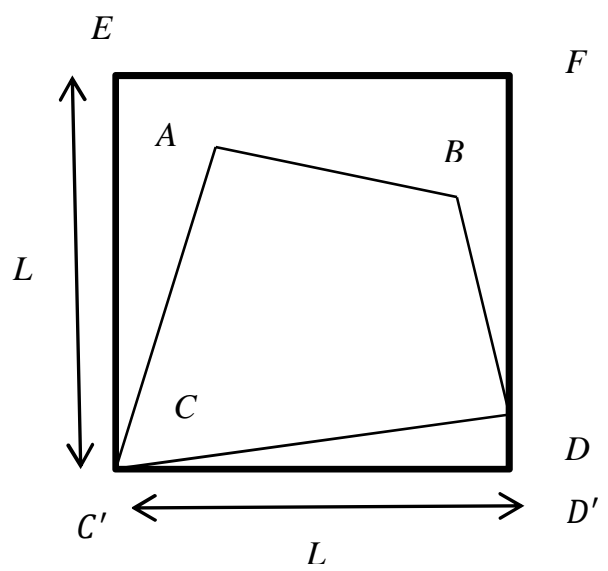

Fig.1 The correspondence between the coordinates of the distorted contour vertices and the coordinates of the standard contour vertices after correction

Step 6: Substitute the four pairs of coordinates into the coordinate transformation equation:

$$
\begin{aligned}
& x_{s}^{\prime}=k_{1} x_{s}+k_{2} y_{s}+k_{3} x_{s} y_{s}+k_{4} \\
& y_{s}^{\prime}=k_{5} x_{s}+k_{6} y_{s}+k_{7} x_{s} y_{s}+k_{8}
\end{aligned}
$$

In the above formula, $x_{s}^{\prime}, y_{s}^{\prime}$ are the standard point's abscissa and ordinate in the corrected image while $x_{s}, y_{s}$ are the corresponding point's abscissa and the ordinate in the distorted image. We get eight equations as shown in the formula (8), solve them, we obtain the correction matrix $k=$ $\left[k_{1}, k_{2}, k_{3}, k_{4}, k_{5}, k_{6}, k_{7}, k_{8}, 1\right]^{T}$. 


$$
\left[\begin{array}{ccccccccc}
x_{a} & y_{a} & x_{a} y_{a} & 1 & 0 & 0 & 0 & 0 & -x_{e} \\
0 & 0 & 0 & 0 & x_{a} & y_{a} & x_{a} y_{a} & 1 & -y_{e} \\
x_{b} & y_{b} & x_{b} y_{b} & 1 & 0 & 0 & 0 & 0 & -x_{f} \\
0 & 0 & 0 & 0 & x_{b} & y_{b} & x_{b} y_{b} & 1 & -y_{f} \\
x_{c} & y_{c} & x_{c} y_{c} & 1 & 0 & 0 & 0 & 0 & -x_{c}^{\prime} \\
0 & 0 & 0 & 0 & x_{c} & y_{c} & x_{c} y_{c} & 1 & -y_{c}^{\prime} \\
x_{d} & y_{d} & x_{d} y_{d} & 1 & 0 & 0 & 0 & 0 & -x_{d}^{\prime} \\
0 & 0 & 0 & 0 & x_{d} & y_{d} & x_{d} y_{d} & 1 & -y_{d}^{\prime}
\end{array}\right]\left[\begin{array}{c}
k_{1} \\
k_{2} \\
k_{3} \\
k_{4} \\
k_{5} \\
k_{6} \\
k_{7} \\
k_{8} \\
1
\end{array}\right]=0
$$

We save the resulting matrix in the application database. The specific preservation method is as follows: each shooting height $h d$ corresponds to a data table, take the height $h d$ as the table index; each record in the data sheet stores the angle information of the $\mathrm{y}$ and $\mathrm{x}$ axis of the cell phone and the image correction matrix $k$ in the corresponding case, the rotation angle of the $\mathrm{y}$ and $\mathrm{x}$ axis is the index of each record, that is, the secondary index or the internal index in the table.

Step 7: Keep the phone's height constant, rotate the phone, and change the rotation angle of the y axis and $\mathrm{x}$ - axis, such as the $\mathrm{y}$ - axis rotation angle of 30 degrees and the $\mathrm{x}$ - axis rotation angle of 1 , the $\mathrm{y}$ - axis rotation angle of 30 degrees and the $\mathrm{x}$ - axis rotation angle of $2 \ldots$ etc. After selecting an angle combination, repeat 3 - 6 times until the correction matrix is obtained with all desired angles, and store them in the online database.

The selection mode of the angle combination: a lot of experiments show that when pedestrians play their phones while walking, $\mathrm{y}$ - axis of the phone rotates around $\mathrm{x}$ - axis in the range of 12 degrees to 40 degrees; when the right hand holds the phone, $\mathrm{x}$ - axis rotates around $\mathrm{y}$ - axis in the range of 0 to 10 degrees, when the left hand holds, the angel range is - 10 degrees to 0 degrees.

When shooting the standard test image, the rotation angle interval of the $\mathrm{y}$ - axis and the $\mathrm{x}$ - axis is one degree, for example, the $\mathrm{y}$ - axis rotation angle is 30 degrees and $\mathrm{x}$ - axis rotation angle is 1 , the $\mathrm{y}$ - axis rotation angle is 30 degrees and $\mathrm{x}$ - axis rotation angle is 2 degrees ... and so on, other angle combinations can be obtained. Shoot a standard test image for each combination, and then calculate the corresponding image correction matrix and store them in the data table with the method given above.

Use Correction Matrix to Correct Distorted Images in Real Time. When the phone application starts for the first time, users input their height $h u$ and arm length $h f$. The program will obtain an approximate shooting height $h d$ as an index key using formula (9), and index the corresponding data table, then download the correction matrix data from the online database, and store the data in the local storage space of the mobile phone application. The method of obtaining the approximate shooting height $h d$ is as follows: set the height of the user as $h u$, the approximate height of the waist is $h e=0.615 \times h u$, the length of the forearm is $h f$, calculate the height of the mobile camera by the following formula:

$$
h d=h e+\sin (\pi / 6) \times h f
$$

The direction sensor of mobile phone ${ }^{[8]}$ collects the angle that $\mathrm{y}$ - axis of the mobile phone rotating around the $\mathrm{x}$ - axis and the angle that $\mathrm{x}$ - axis rotating around the $\mathrm{y}-$ axis, the two angles are zero on the horizontal plane, and the sensor will collect these two angels in real time at a specific frequency according to the precision of the application ${ }^{[9]}$. Sensor data changes very fast, this paper collects five sensor data immediately after collecting a frame picture, and uses the average of these five data as the final angle value, then use it to index the corresponding correction matrix in the datasheet that has been downloaded to the local data table. The sensor collects images twice a second, and will start the follow-up treatment immediately. 
For the pixel point $i$ in the image captured in real time, perform coordinate conversion according to the following formula (10):

$$
\left(x_{s}^{\prime}, y_{s}^{\prime}\right)=T\left(x_{s}, y_{s}\right)
$$

$T$ is the spatial transformation relationship realized by formula (6) and formula (7). Thus, the complete spatial transformation relationship is obtained. Then, the fast correction and gray value reconstruction of a frame image can be accomplished by using this spatial transformation relation and bilinear interpolation method.

Experimental Analysis. According to the proposed algorithm, a large enough standard squared paper is used to take the standard test image, and then calculate the distortion correction matrix based on the standard test image. The calculation in this test requires mobile sensor coordinate system and digital image coordinate system. The results are as follows:

Experiment 1: the shooting height is $110 \mathrm{~cm}$, the rotation angle of $\mathrm{y}-$ axis is 30 degrees, and the rotation angle of $\mathrm{x}-$ axis is 0 .
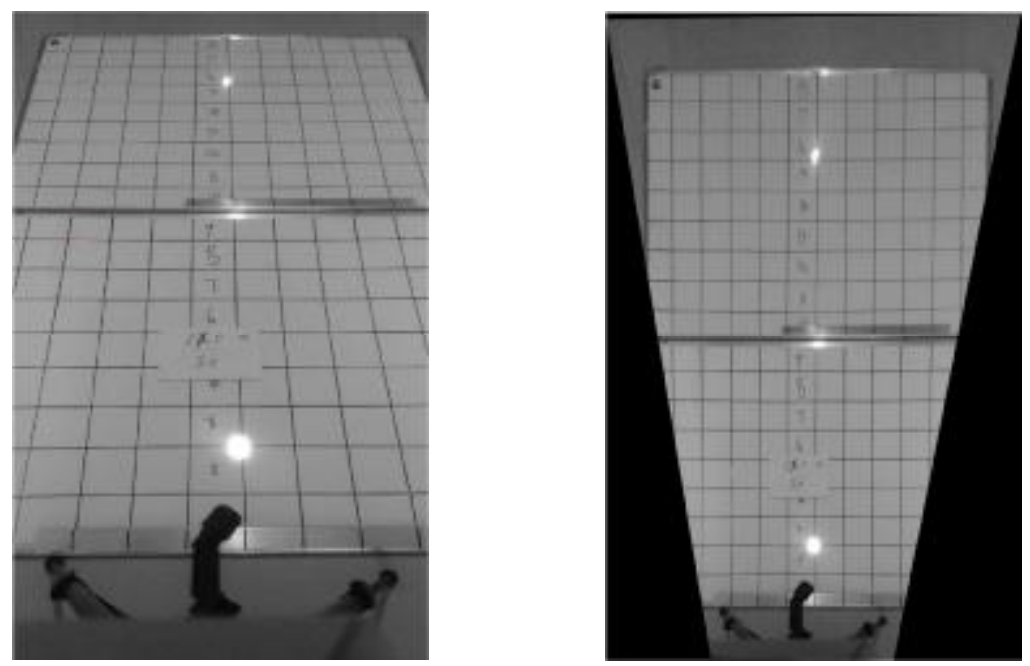

Fig.2 The standard test image and its corrected image in experiment 1
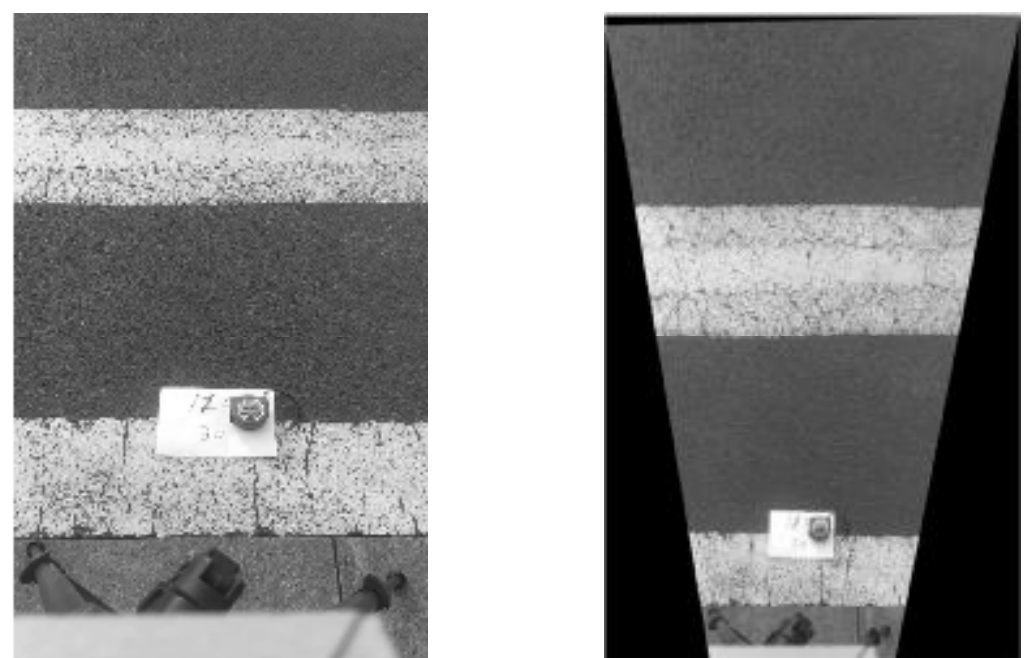

Fig.3 The original road image and the image after correction using the corresponding correction matrix in experiment 1

Experiment 2: The shooting height is $120 \mathrm{~cm}$, the rotation angle of $\mathrm{y}-$ axis is 30 degrees, and the rotation angle of $\mathrm{x}$ - axis is 0 degrees. 

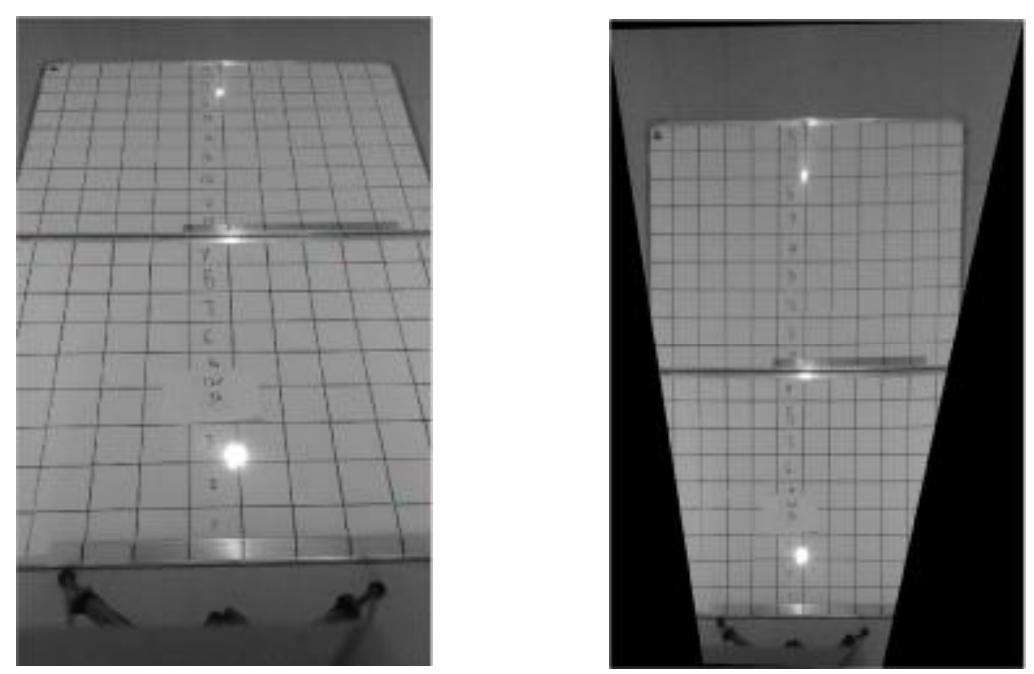

Fig.4 The standard test image and its corrected image in experiment 2
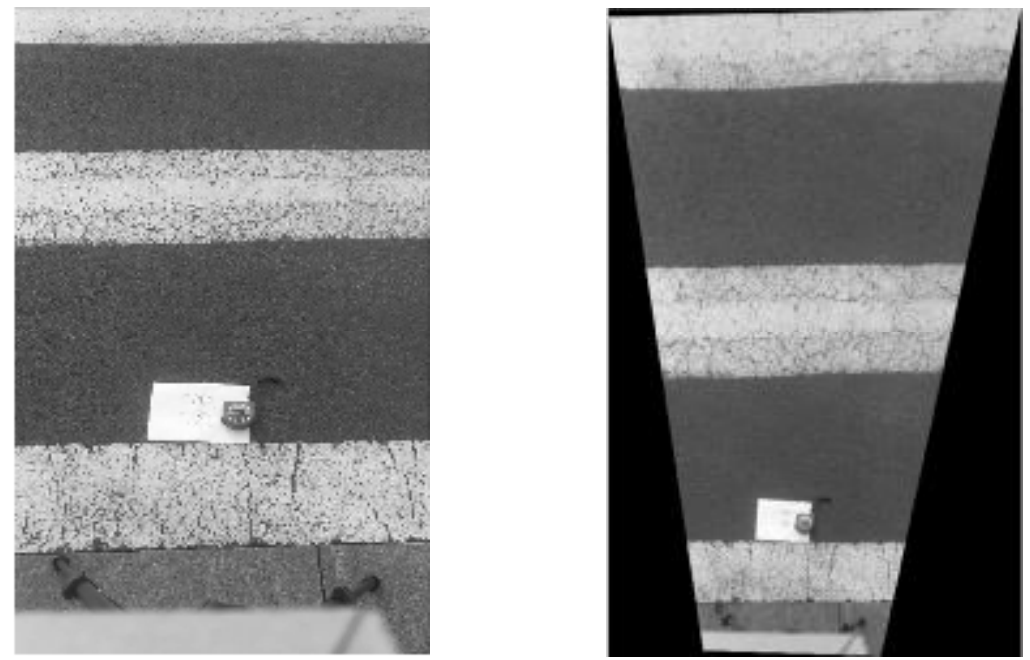

Fig.5 The original road image and the image after correction using the corresponding correction matrix in experiment 2

The angle information used in the experiment is all obtained in real time using the direction sensor of the smart phone. From the experimental results, we can see that the algorithm proposed in this chapter can correct the distorted images fast, and achieve the expected functional requirements. Because the algorithm separates the complex computation of correction matrix from the image correction process, instead of completing it separately in advance. The subsequent correction algorithm directly invokes the existing correction matrix to correct the distorted images, thus the operation time of the correction algorithm is reduced to a great extent, and the real-time performance of the image correction is ensured. Experiments on Huawei's G7 mobile platform show that, in the case of the corresponding correction matrix, the average correction time of a distorted image with a resolution of $721 * 1280$ is a few tens of milliseconds. In practice, the consuming time of the algorithm can be further reduced by reducing the resolution of the image. Therefore, the algorithm proposed in this chapter can meet the requirements of real-time performance, and has good application value.

\section{Conclusions}

This paper proposes a real-time image distortion correction method based on correction matrix, which can quickly correct the image of trapezoidal distortion, and has good practical application value, can be applied to "Phubbing" safety protection. As chips become more powerful, the computing ability of the artificial intelligent mobile computing platform will also greatly improve, we can expect to calculate the correction matrix in real time on the mobile phone, and use it to do 
distortion correction, which will improve the accuracy and real-time performance of image processing, and can also provide users with more real-time security protection.

\section{References}

[1] Bogdan Kwolek, Michal Kepski. Human fall detection on embedded platform using depth maps and wireless accelerometer [J]. Computer Methods and Programs in Biomedicine. 2014

[2] Heba Aly, Anas Basalamah,Moustafa Youssef. Robust and ubiquitous smartphone-based lane detection [J]. Pervasive and Mobile Computing, 2016, 26.

[3] Zhangsen, Zhaoqunfei, Yejianke. A Method of Auto-correcting Geometrical Distortion of Digital Images [J]. Mechatronics.2007

[4] Kannala Juho, Brandt Sami S. A Generic Camera Model and Calibration Method for Conventional, Wide-Angle, and Fish-Eye Lenses [J]. IEEE Transactions on Pattern Analysis and Machine Intelligence, 2009， 28 (8): 1335-1340.

[5] Digital Image Processing. Gonzalez Rafael C, Woods Richard E. 2007

[6] Zhang Yu. Research on trapezoidal distortion correction algorithm and application of video real time correction [D]. Anhui University, 2014.

[7] J. Herráez, J.L. Denia, P. Navarro, J. Rodríguez, M.T. Martín. Determining image distortion and pbs (point of best symmetry) in digital images using straight line matrices [J]. Measurement, 2016.

[8] Zhao Hong, Guo Li. Space coordinate transformation algorithm for the acceleration sensor data of mobile phone [J]. Computer application, 2016, 36 (02): 301 - 306.

[9] Wagner Daniel, Reitmayr Gerhard, Mulloni Alessandro, Drummond Tom, Schmalstieg Dieter .Real-time detection and tracking for augmented reality on mobile phones. IEEE Transactions on Visualization and Computer Graphics. 2010 